\section{The forgotten fallopian tube}

\section{Johannes Dietl and Jörg Wischhusen}

In David D. L. Bowtell's recent Opinion article (The genesis and evolution of highgrade serous ovarian cancer. Nature Rev. Cancer 10, 803-808 (2010)) ${ }^{1}$, a considerable body of evidence is presented which further supports the central role of the fallopian tube in the pathogenesis of this disease. The hypothesis that a substantial proportion of high-grade serous ovarian cancers (HG-SOC) arise from the distal fallopian tube has also been proposed by several other unrelated investigations $s^{2-9}$. We think that these findings made by molecular geneticists and pathologists should now be translated into clinical practice. The immediate consequence for preventive medicine would be the easy-to-carry out simultaneous removal of the fallopian tubes during surgery, such as hysterectomy (removal of the uterus) or sterilization (coagulation or partial resection of the fallopian tubes). Sparing the fallopian tubes during hysterectomy provides no known physiological benefits, as the remaining tubes are completely devoid of any function. As the blood supply to the ovaries is secured through vessels from the infundibulopelvic ligament, it is not affected when hysterectomy is combined with bilateral removal of the fallopian tubes (salpingectomy). The hormone profile is not altered by salpingectomy, even several months after surgery ${ }^{10}$. The blind ending remnants of the tubes can provide a nidus for bacteria and thereby increase infectious morbidity and tubal pathology that can require further surgery ${ }^{11}$. However, in the light of recent findings, as discussed in Bowtell's article, the strongest reason for bilateral salpingectomy or fimbriectomy along with hysterectomy or sterilization is the potential role of the fallopian tube in the pathogenesis of HG-SOC. This disease accounts for the largest proportion of ovarian cancers and for about two-thirds of ovarian cancer-related deaths.

Such a revised strategy would clearly represent a paradigm change in preventive surgery. Until recently, all debates on the aetiology and prevention of ovarian cancer have focussed on the ovaries, whereas the fallopian tube was largely forgotten. Now, it is becoming clear that clinicians must pay due attention to the fallopian tube. Thus, simultaneous risk-reducing salpingectomy should become the new standard whenever a hysterectomy needs to be carried out.

We also think that, based on the discussions above, the need for bilateral removal of the ovaries (oophorectomy) should be reassessed on an individual basis, especially as this procedure has immediate consequences for the patient. Alarming figures from the United States show that bilateral oophorectomy is carried out in $18 \%$ of American women aged between 18 and 44 years old who undergo hysterectomy for benign diseases. In the age group of 45 to 64 years old, this proportion can be as high as $76 \%{ }^{12}$. Large retrospective studies have shown that a surgical menopause increases long-term risks for cardiovascular, psycho-sexual and cognitive dysfunctions ${ }^{12}$. Bilateral salpingectomy, by contrast, is not associated with these risks or other major side effects, but may nevertheless be most effective in reducing or even preventing the risk for HG-SOC. Thus, whenever a hysterectomy needs to be carried out, simultaneous risk-reducing salpingectomy can generally be recommended, whereas the case for oophorectomy should be made only after serious consideration. Moreover, patients who do not want to undergo preventive salpingectomy and oophorectomy in spite of a known familial risk of ovarian cancer could be offered salpingectomy only. Based on recent results, this might afford a high degree of protection with a minimal affect on quality of life.

Johannes Dietl and Jörg Wischhusen are at the Department for Obstetrics and Gynaecology, JuliusMaximilians-University Würzburg, School of Medicine, Josef-Schneider-Str. 4, 97080 Würzburg, Germany. Correspondence to J.D e-mail: Dietl J@klinik.uni-wuerzburg.de doi: 10.1038/nrc2946-c1

1. Bowtell, D. D. L. The genesis and evolution of high-grade serous ovarian cancer. Nature Rev. Cancer 10, 803-808 (2010).

2. Kindelberger, D. W. et al. Intraepithelial carcinoma of the fimbria and pelvic serous carcinoma: evidence for a causal relationship. Am. J. Surg. Pathol. 31, 161-169 (2007)

3. Carlson, J. W. et al. Serous tubal intraepithelial carcinoma: its potential role in primary peritoneal serous carcinoma and serous cancer prevention. J. Clin. Oncol. 26, 4160-4165 (2008).

4. Przybycin, C. G., Kurman, R. J., Ronnett, B. M., Shih, I.-M. \& Vang, R. Are all pelvic (nonuterine) serous carcinomas of tubal origin? Am. J. Surg. Pathol. 34, 1407-1416 (2010).

5. Chen, E. Y. et al. Secretory cell outgrowth, PAX2 and serous carcinogenesis in the fallopian tube. J. Pathol. 222, 110-116 (2010).

6. Roh, M. H. et al. High-grade fimbrial-ovarian carcinomas are unified by altered p53, PTEN and PAX expression. Mod. Pathol. 23, 1316-1324 (2010).

7. Kurman, R. J. \& Shih, I.-M. The origin and pathogenesis of epithelial ovarian cancer: A proposed unifying theory. Am. J. Surg. Pathol. 34, 433-443 (2010).

8. Kuhn, E. et al. Shortened telomeres in serous tubal intraepithelial carcinoma: an early event in ovarian high-grade serous carcinogenesis. Am. J. Surg. Pathol. 34, 829-836 (2010).

9. Herrington, S. S. \& McCluggage, W. G. The emerging role of the distal fallopian tube and p53 in pelvic serous carcinogenesis. J. Pathol. 220, 5-6 (2010).

10. Sezik, M., Ozkaya, O., Demir, F., Sezik, H. T. \& Kaya, $\mathrm{H}$. Total salpingectomy during abdominal hysterectomy: effects on ovarian reserve and ovarian stromal blood flow. J. Obstet. Gynecol. Res. 33, 863-869 (2007)

11. Ghezzi, F., Cromi, A., Siesto, G., Bergamini, V., Zefiro, F. \& Bolis, P. Infectious morbidity after total laparoscopic hysterectomy: does concomitant salpingectomy make a difference? BJOG 116 , 589-593 (2009)

12. Hickey, M., Ambekar, M. \& Hammond, I. Should the ovaries be removed or retained at the time of hysterectomy for benign disease? Hum. Reprod. Update 16, 131-141 (2010).

Competing interests statement

The authors declare no competing financial interests. 